\title{
Spectral classification systems and photometry needed in calculations for atmospheric refraction
}

\author{
V. Malyuto ${ }^{1,2}$ and M. Meinel ${ }^{2}$ \\ 1 Tartu Observatory, 61062 Tartumaa, Tõravere, Estonia \\ e-mail: valeri@aai.ee \\ 2 Dresden University of Technology, Lohrmann Observatory, Mommsenstrasse 13, 01062 Dresden, Germany \\ e-mail: meinel@kobsw4.geo.tu-dresden.de
}

Received November 3, 1999; accepted January 5, 2000

\begin{abstract}
In ground-based measurements of exact positions of celestial objects on the sky the astronomical atmospheric refraction should be taken into account. In our calculations for refraction we use the Stone code (Stone 1996) where the mean refraction is computed by integrating numerically across the passband with Simpson's Rule. The code has been modified for the case of the $10^{\prime \prime}$ LX200 telescope installed at the Lohrmann Observatory in Dresden. Tabulations of stellar spectral energy from Sviderskiene (1988) together with the quantum efficiency of the CCD detector being used, are applied. We have estimated the accuracies of stellar classification required to achieve the desirable accuracies in calculated refraction, the influence of ground-based atmospheric parameters has been discussed too. Some examples of the spectral classification systems which may provide the required classification accuracies, are given. The special case of computing differential refraction has also been considered. We justify the usefulness of photometric $V-R$ and $V-I$ indices in calculating refraction. Observations are planned to compare calculated and true refraction.
\end{abstract}

Key words: atmospheric effects — stars: fundamental parameters - techniques: miscellaneous

\section{Introduction}

Knowledge of exact positions of celestial objects on the sky (solar system bodies, stars, galaxies) is important in many fields of astronomy (astrometry, celestial mechanics, astrophysics, stellar astronomy, galactic studies). The most exact data are being obtained from the extraterrestrial platforms which provide much better astrometric

Send offprint requests to: V. Malyuto accuracy than any ground-based observation. The number of astrometric measurements from space grows very fast, parallel to their accuracy $(10-1000$ microarcsecs $)$ and limiting magnitudes $(10-20 \mathrm{mag})$ in the most ambitious HIPPARCOS, DIVA and GAIA projects. However, these projects are expensive, the space missions are rather rare and only last few years. The ground-based observations may be performed practically at any time and retain their significance, especially for fast-moving (asteroids) and/or short-living (comets) objects.

We may expect that in future a dense network of accurate extraterrestrial positions of stars will be created, and ground-based positions will be performed mainly differentially (relative to the positions of the reference stars measured from space). The accuracy of such ground-based relative astrometry can be rather high and may even approach the accuracy of extraterrestrial positions for the reference stars. As an example we mention some groundbased interferometric programs, currently being planned to perform relative astrometry in very small fields to the level of 100 microarcseconds (Gubler \& Tytler 1998). According to Kovalevsky (2000) ground-based astrometric observations should concentrate now on small fields and relative motions. Among present-day astrometric tasks, measurements of double stars and positions of minor components of the solar system (moons, asteroids) are mentioned.

To determine the exact positions of celestial objects it is necessary to include all the displacements and motions involved in the reference system. Among these the refraction should be taken into account. By definition, atmospheric refraction, or simply refraction $R$, is

$R=z-z_{0}$,

where $z_{0}$ is the apparent zenith distance on the ground, $z$ is the zenith distance that would have been measured if there were no atmosphere. 
A real source has a certain spectral energy distribution and the terrestrial atmosphere acts on it like a prism changing the zenith distance of a source. The effect is diminishing from blue to red, and therefore the point source is being transformed into a rainbow. If the light refraction (as a function of wavelength) is known, the exact position of the source may be calculated as the source's light gravity center by convolution of the refraction with the following relevant functions: intrinsic spectral energy distribution caused by interstellar reddening, atmospheric transmission, transmission of the telescope in combination with the quantum efficiency of the detector.

The most comprehensive analysis of the problem has been performed by Stone in his two papers (Stone 1984, 1996). He has applied an approach in defining the refraction with Danjon's formula and Owens (1967) refractivities which produces a very good approximation for zenith distances $z_{0} \leq 75^{\circ}$. It only requires knowledge of the meteorological conditions at the observing site (groundbased atmospheric temperature, pressure, relative humidity) and the apparent zenith distance of the object being observed. The appropriate analytical expressions are used to calculate $R(\lambda, z)$. The published spectral energy distributions of stars of different spectral types and luminosity classes together with the functions just mentioned in the previous paragraph have been applied. It has been found that the calculated dependencies of stellar positions on spectral types agree extremely well with the observations. The color effect is rather small but significant: for example, the refraction changes by about 0.1 arcsec if one goes from spectral type $\mathrm{B}$ to $\mathrm{M}$ at a zenith distance of $60^{\circ}$. However, one may suspect that this effect should be much larger in case of using detectors with wider spectral sensitivity range (passband). Schildknecht (1994) has used a similar approach in calculating refraction but the passband is $4700-8800 \AA$. Thus the respective variation with spectral type $(\mathrm{B}-\mathrm{M})$ reaches 0.5 arcsec at the zenith distance of $60^{\circ}$. Schildknecht has not discussed the variations with luminosity class and interstellar reddening.

The recent paper of Gubler \& Tytler (1998) deals with the refraction calculations based on a more detailed atmospheric model and using a numerical integration by iterations. Some simplified and faster methods have been tried out with the result that they agree well for any reasonable set of atmospheric parameters. In these calculations, the assumption has been made that the star radiates as a blackbody at a temperature equal to the surface temperature of the star. Another simplifying assumption is that atmospheric transmission, filter transparency and detector efficiency are constant values $(=1)$ in the filter bandpass. Authors have discussed how accurately the relevant quantities (ground-level atmospheric temperature, pressure, relative humidity, as well as temperatures of two stars) must be measured to limit the errors in the differential refraction to 10 microarcsec or less per input parameter. The most stringent of these requirements is that the stellar surface temperature should be known accurately $(\sim 100 \mathrm{~K})$ if the star is cool.

Determining the temperatures of stars from their spectral or photometric data is a task of stellar classification. For classification we need a certain information on stellar spectral energy distributions (low resolution spectra or photometric measurements in filters) in order to apply appropriate classification methods (systems) to these data. In general, any spectral feature depends on three basic stellar physical parameters: effective temperature, gravity and metal abundance. Classification methods use available spectral information to assign these values (or some relevant parameters) to a star. A conventional approach is to assign MK spectral types and luminosity classes (or absolute magnitudes); the latter are closely connected with the basic physical parameters. The available calibrations "spectral type versus effective temperature" and "luminosity class versus gravity" may be applied to MK data if necessary. Metal abundances are characterized by $[\mathrm{Fe} / \mathrm{H}]$ values.

As a typical example we mention here the photometric classification method elaborated by Vilnius astronomers (photoelectric measurements in some filters are required). There, stars brighter $14^{\mathrm{m}}$ (with a $1 \mathrm{~m}$ telescope) are routinely classified (Straizys 1992). MK spectral types, absolute magnitudes and $[\mathrm{Fe} / \mathrm{H}]$ values are defined. As to spectral classification methods, Houck (1994) performs the Michigan Survey (being completed in 7 volumes by 2004) based on photographic objective prism spectra $2 \AA$ resolution) and providing MK types and luminosity classes (by visual inspection) for many thousands of stars over the sky. Some recent spectral studies provide quantitatively the MK types and luminosity classes based on $10-15 \AA$ resolution spectra, diode-array and CCD detectors are being used (Malyuto \& Schmidt-Kaler 1997; Malyuto et al. 1997; Weaver \& Torres-Dodgen 1997).

In the present paper we analyze the possibility of performing high accuracy refraction measurements with the $10^{\prime \prime}$ LX200 telescope installed at the Lohrmann Observatory in Dresden. The Stone (1996) code has been applied to calculate the refraction with the use of the published representative stellar spectral energy distributions of different spectral types and luminosity classes (Sviderskiene 1988) at different degrees of interstellar reddening; appropriate meteorological parameters, atmospheric transmission, transmission of the telescope in combination with the quantum efficiency of the CCD detector have been involved. We discuss which stellar parameters are useful and how accurate the stellar input and ground-based atmospheric parameters should be to provide accurate calculations for atmospheric refraction. We describe some stellar spectral classification and photometric systems which may provide the necessary accuracies of stellar parameters. Future improvements of the systems are discussed. 


\section{Method of calculations and data sources}

Dr. R. Stone (U.S. Naval Observatory) has kindly sent us the code developed for computing refraction in his SDSS survey (Stone 1984, 1996). He considers this approach to be very simple (only analytical expressions are used), fast and also very accurate: for zenith distances under $z_{0} \leq 65^{\circ}$ the error in the computed refraction is 0.01 arcsec and smaller.

The refraction, really observed at a telescope, is so called mean refraction; it can be calculated by

$R_{\mathrm{m}}(z)=\frac{\int_{\lambda_{\min }}^{\lambda_{\max }} R(\lambda, z) S(\lambda) E(\lambda) A(\lambda, z) L(\lambda) D(\lambda) \mathrm{d} \lambda}{\int_{\lambda_{\min }}^{\lambda_{\max }} S(\lambda) E(\lambda) A(\lambda, z) L(\lambda) D(\lambda) \mathrm{d} \lambda}$,

where $R_{\mathrm{m}}(z)$ is the mean refraction at zenith distance $z, R(\lambda, z)$ is the selective refraction for monochromatic light at wavelength $\lambda ; S(\lambda)$ is the spectral energy distribution for a star being observed; $E(\lambda)$ is the transmittance of interstellar matter for the color excess $E(B-V)$; $A(\lambda, z)$ is the transmission of the atmosphere; $L(\lambda)$ is the transmission of the telescope optics; $D(\lambda)$ is the quantum efficiency of the detector being used. In the Stone code the mean refraction is computed by integrating numerically the Eq. (2) across the passband with Simpson's Rule. This mean refraction corresponds to the light gravity center of the image of a point source and the approach used is the only correct one, at least for broad passbands (Schildknecht 1994).

We apply the Stone code to analyse the data which are obtained with the $10^{\prime \prime}$ LX200 telescope installed at the Lohrmann Observatory in Dresden. The transmission curve of the telescope optics and the quantum efficiency of the CCD detector (KAF-1600 device) have been received from manufacturers. No filters are installed. The empirical curve of the transmission of the atmosphere taken by Stone from Palomar has been replaced by the typical transmission function of the Earth's atmosphere at the zenith, given in the Straizys's monograph (Straizys 1992).

We use the more recent, compared to Stone (1984, 1996), stellar spectral energy distributions for selected spectral types from $\mathrm{O}$ to $\mathrm{M}$ as well as for different luminosity classes from I to $\mathrm{V}$, as given by Sviderskiene (1988). These tabulations have been commented by Pickles (1998): "its scope, consistency and accuracy of energy distribution make it an ideal check on all other input spectra for flux calibration and accuracy". To calculate interstellar absorption as a function of a wavelength $\lambda$, we use the same approach as in the Stone code (based on the extinction law, tabulated by Allen 1966).

\section{Results of refraction calculations and their analysis}

For our calculations we have adopted the following values of meteorological conditions at the observing site: atmo- spheric temperature $7^{\circ} \mathrm{C}$, pressure $745 \mathrm{~mm} \mathrm{Hg}$, dew point $40^{\circ} \mathrm{F}$. In the present study all calculations are performed at the zenith distance of $60^{\circ}$. Results may be compared with other data presented for the same zenith distance in other similar studies (e.g. Schildknecht 1994).

We have calculated the mean refraction with Eq. (2) for stellar spectral energy distributions of every spectral type separately for three representative luminosity classes: dwarfs (luminosity class V), giants (luminosity class III) and supergiants (luminosity class I) as well as at different degrees of interstellar reddening $(E(B-V)=0.0,0.5,1.0$, $2.0,3.0,4.0)$.

In Fig. 1 the dependence of the calculated refraction on MK spectral types is given for dwarfs. In the paper of Schildknecht (1994) these data were calculated for the same zenith distance and are given in tabulated form, therefore the direct comparison is possible. As the data of Schildknecht are given differentially, we have added our data at G0 to his zero-point. The dependencies are in good agreement, taking into account that the different spectral energy distributions, CCD detectors and telescope optics were involved. But our dependence in Fig. 1 shows the gentlest sloping for late type stars while in the dependence of Schildknecht this is the case for early type stars. In their analysis of the dependence of differential refraction on stellar temperatures Gubler \& Tytler (1998) have found that for cool stars the dependence shows the gentlest sloping, in agreement with our data. Schildknecht (1994) as well as Gubler \& Tytler (1998) have not discussed any luminosity and interstellar reddening effects.

Accurate estimates of observational errors in determining star positions have been made by Stone et al. (1996) with the FASTT telescope. After careful reductions and analysis the accuracy of about 0.15 arcsec has been achieved in their paper ( 0.14 and 0.17 arcsec, respectively in right ascension and declination for a single observation). It is desirable to calculate refraction with better accuracy (by about one order) than the accuracy of observed stellar positions.

The errors in calculated refraction may arise from incomplete knowledge of stellar and ground-based atmospheric parameters as well as inherent limitations of the refraction model. To every stellar spectral energy distribution used in the analysis we have attributed the $T_{\text {eff }}$ values with the use of the calibration "Spectral type versus $T_{\text {eff }}$ " of Schmidt-Kaler (1982). The dependence of the calculated refraction on $T_{\text {eff }}$ for dwarfs is given in Fig. 2. We see that the slope of this dependence is changing with temperature. We have divided the data in four groups according to effective temperatures $(30000-10500 \mathrm{~K}, 9500-8700 \mathrm{~K}$, $8200-4900 \mathrm{~K}, 4700-4100 \mathrm{~K}$ and $3800-2900 \mathrm{~K}$, which correspond to spectral types B, A0-A3, A5-K2, K3-K7 and $\mathrm{M}$, respectively). The slopes of the dependence within each group are approximately linear. From these slopes we have estimated how accurate the $T_{\text {eff }}$ values should be to achieve the desirable accuracies of $0.02,0.05$ and 


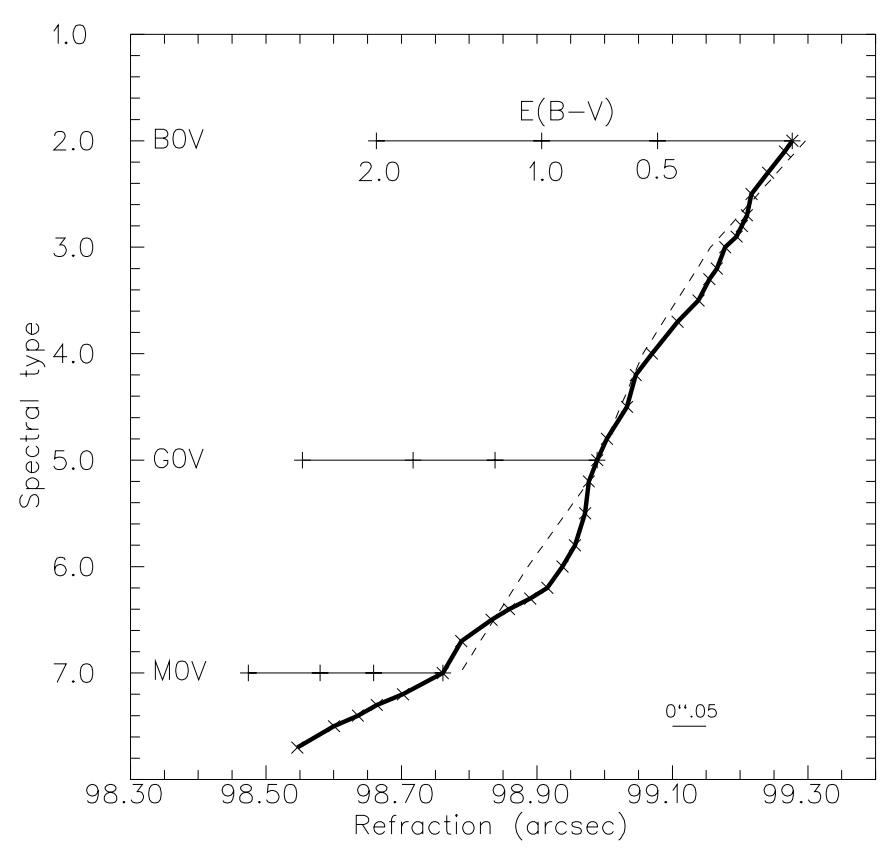

Fig. 1. The dependence of the calculated refraction on MK spectral types for dwarfs. The continuous spectral codes for discrete spectral types were introduced as follows: $\mathrm{B} 0=2.0, \mathrm{~A} 0=3.0$, $\mathrm{F} 0=4.0, \mathrm{G} 0=5.0, \mathrm{~K} 0=6.0$ and $\mathrm{M} 0=7.0$. The reddenings (thin lines) are shown for B0, G0 and M0 dwarfs at different $E(B-V)$. The dependence from Schildknecht (1994) is imposed by the dashed line. One desirable error in refraction $(0.05$ arcsec $)$ is indicated. All calculations have been made at the zenith distance of $60^{\circ}$

Table 1. Accuracies in $T_{\text {eff }}[\mathrm{K}]$ required to achieve the desirable accuracies per parameter in calculated refraction for dwarfs

\begin{tabular}{l|rrrrr}
\hline Accuracy in & \multicolumn{5}{|c}{ Accuracy in $T_{\text {eff }}$} \\
refraction [as] & $\mathrm{B}$ & $\mathrm{A} 0-\mathrm{A} 3$ & $\mathrm{~A} 5-\mathrm{K} 2$ & $\mathrm{~K} 3-\mathrm{K} 7$ & $\mathrm{M}$ \\
\hline 0.02 & 4550 & 690 & 300 & 140 & 80 \\
0.05 & 11370 & 1730 & 740 & 340 & 200 \\
0.10 & 22740 & 3470 & 1480 & 680 & 410 \\
\hline
\end{tabular}

0.1 arcsec in calculated refraction, respectively. These estimates for the accuracy required for $T_{\text {eff }}$ (based in Fig. 2) are presented in Table 1 . The respective estimates for the accuracy required for spectral types (based in Fig. 1) are presented in Table 2.

The most stringent requirement to accuracy of $T_{\text {eff }}$ refers to $\mathrm{K}-\mathrm{M}$ dwarfs. We conclude from the first columns of Tables 1 and 2 that we should have the accuracies of $80-140 \mathrm{~K}$ in $T_{\text {eff }}$ or $0.6-0.8$ in spectral subtype respectively in order to achieve an accuracy of 0.02 arcsec in calculated refraction. The corresponding analysis for other luminosity classes (giants and supergiants) leads us to about the same conclusions.

The results of Tables 1 and 2 should be compared with the real accuracies of spectral classification achieved with available spectral classification methods. A system of spec-

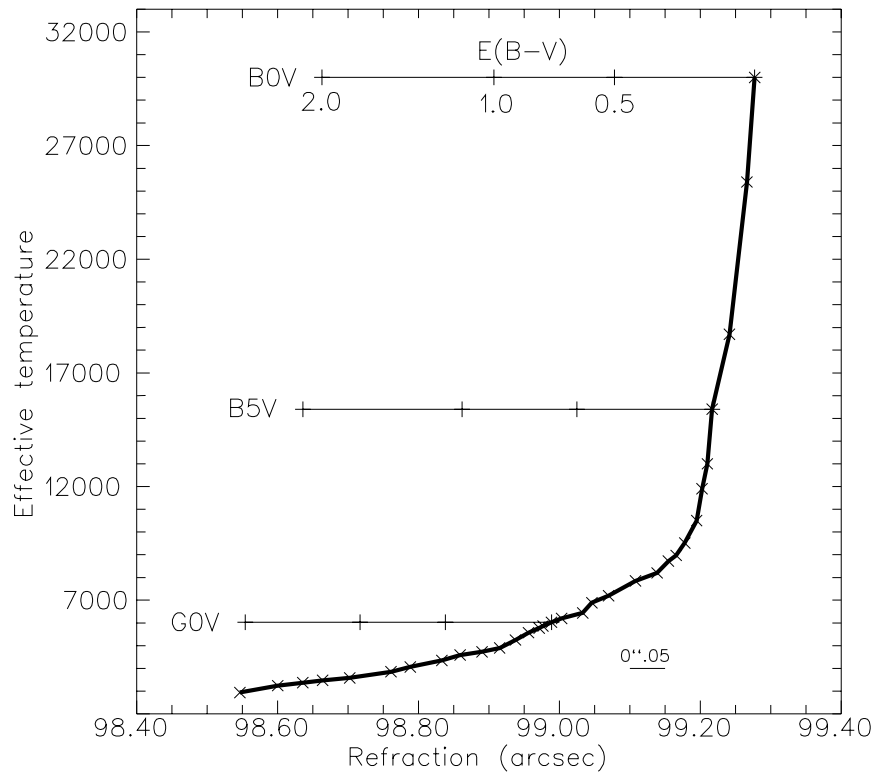

Fig. 2. The dependence of the calculated refraction on $T_{\text {eff }}$ for dwarfs. The reddenings (thin lines) are shown for B0, B5 and G0 dwarfs ( $T_{\text {eff }}=3000,5860$ and $3580 \mathrm{~K}$, respectively) at different $E(B-V)$. One desirable error in refraction $(0.05$ arcsec $)$ is indicated. All calculations have been made at the zenith distance of $60^{\circ}$

Table 2. Accuracies in spectral subtypes required to achieve desirable accuracies per parameter in calculated refraction for dwarfs

\begin{tabular}{l|rrrr}
\hline Accuracy in & \multicolumn{4}{|c}{ Accuracy in spectral subclasses } \\
refraction [as] & B-G2 & G3-K0 & K2-K7 & M \\
\hline 0.02 & 2.1 & 3.0 & 0.8 & 0.6 \\
0.05 & 5.1 & 7.4 & 1.9 & 1.6 \\
0.10 & 10.3 & 14.8 & 3.9 & 3.2 \\
\hline
\end{tabular}

tral classification for $\mathrm{K}-\mathrm{M}$ stars developed by Malyuto et al. (1997) is based on spectral indices measured with different spectral libraries of about $10 \AA$ resolution (data from photoelectric scanners, diode-array and CCD detectors are involved). The accuracy of classification is 0.6 of spectral subtype which roughly corresponds to $100 \mathrm{~K}$ according to the calibration "Spectral type versus $T_{\text {eff }}$ " of Schmidt-Kaler (1982). This accuracy estimate includes the uncertainty of the original MK classifications from the literature and the accuracy of the method should be better. A more informative approach to classification exhausting the information contained in the spectrum (so called perturbation method) has been developed by Cayrel et al. (1991) and others (Soubiran et al. 1998; Katz et al. 1998) for F5 - K7 stars having $5 \AA$ resolution CCD spectra, which provide the accuracy of $145 \mathrm{~K}$ in $T_{\text {eff }}$, the inhomogeneity of the parameters from the literature is included, too. These two approaches have been successfully applied to stars up to $V=13^{\mathrm{m}}$ and $15^{\mathrm{m}}$, respectively. As to 
photometric classifications, the Vilnius system (Straizys 1992) provides the respective classification accuracy of 0.8 of spectral subtype or about $150 \mathrm{~K}$ for $\mathrm{K}-\mathrm{M}$ stars (the uncertainty of the original MK classifications are included too). Straizys et al. (1998) have calculated the accuracies of some photometric systems from the analysis of the Kurucz model atmospheres for stars earlier M0. The Vilnius system provides the best accuracy (better than $100 \mathrm{~K}$ for $\mathrm{K}$ stars, the corresponding errors of spectral types are from 0.1 to 0.5 of spectral subtypes). From the comparison of these temperature accuracy estimates with the data of Tables 1 and 2 for $\mathrm{K}-\mathrm{M}$ stars we conclude that the accuracy of 0.02 arcsec per $T_{\text {eff }}$ parameter in refraction is achievable with the available spectral and photometric classification systems.

If we agree to be within the larger error budget of 0.05 arcsec per temperature parameter in calculated refraction, we may respectively weaken our requirements to the accuracies of spectral types (it allows to reach fainter stars). For example, judging on Table 2, accuracy of 2 spectral subtypes could then be sufficient. If we increase this error budget to 0.1 arcsec, accuracy of 4 spectral subtypes could be sufficient. In this aspect it is to the point to mention some classification systems providing relatively low classification accuracy (these systems are applicable to faint stars). Some of them are based on photographic objective prism spectra of very low resolution (e.g. Upgren 1962, dispersion of $580 \AA / \mathrm{mm}$, what corresponds to $9 \AA$ resolution, classification accuracy is about 2 spectral subtypes, the limiting magnitude is about $13^{\mathrm{m}}$ with the Hamburg $80 \mathrm{~cm}$ Schmidt telescope). Seitter (1975) has developed spectral classification systems based on photographic objective prism spectra of different low dispersions (645 and $1280 \AA / \mathrm{mm}$, what correspond to 10 and $20 \AA$ resolutions, respectively). Observations have been performed at the Hoher List Observatory of Bonn University, diameter of correcting plate of the Schmidt telescope is $34 \mathrm{~cm}$. The classification accuracies are 1.6 and 3 spectral subtypes, respectively. As an example of very low resolution spectral classification studies, we mention a paper of Nandy et al. (1977) where a description of an objective prism for the $1.2 \mathrm{~m}$ Schmidt telescope is given, dispersion is $2500 \AA / \mathrm{mm}$ (about $40 \AA$ resolution), what provides photographic spectral classification with an accuracy of 5 spectral subtypes, the limiting magnitude of $20^{\mathrm{m}}$ may be even achieved.

Variations of calculated refraction with the spectral luminosity classes should be analysed, too. In Fig. 3 the calculated refraction is given as a function of spectral type for luminosity classes I, III and V (supergiants, giants and dwarfs), respectively. The luminosity effects in refraction seem to be significant, differences between luminosity classes reach 0.06 arcsec for $\mathrm{K}-\mathrm{M}$ stars. Judging on positions of luminosity class lines in Fig. 3 we see that we should only distinguish supergiants from stars of other luminosity classes (giants and dwarfs) for stars of spec-

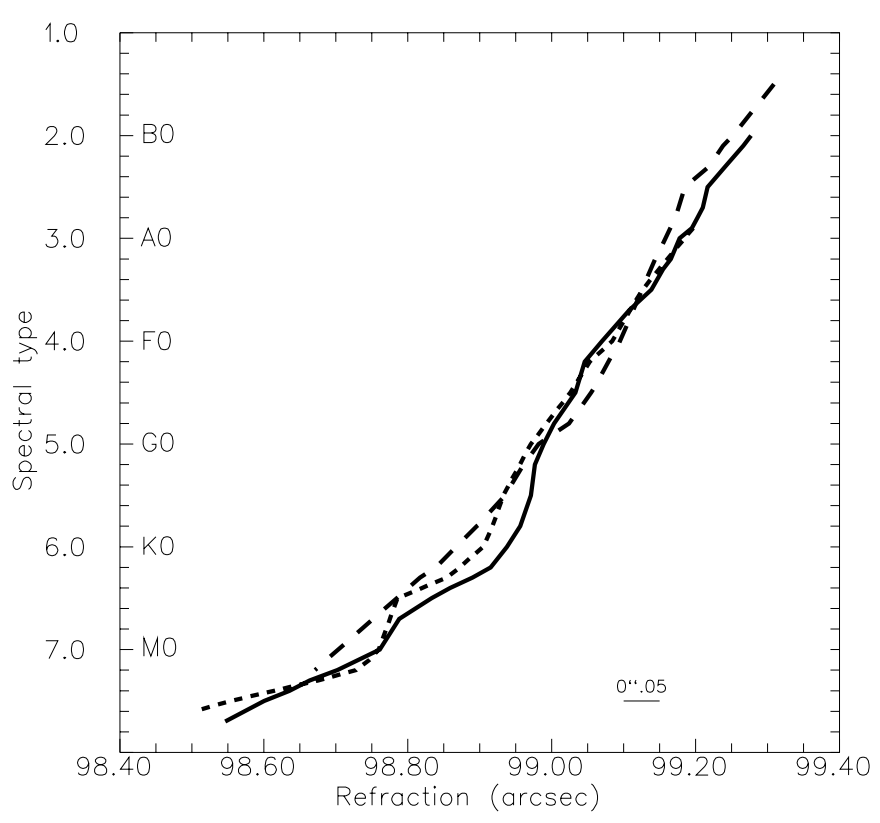

Fig. 3. The dependencies of the calculated refraction on spectral types for different luminosity classes. The solid line corresponds to luminosity class $\mathrm{V}$, the short-dashed line to luminosity class III, the long-dashed line to luminosity class I. One desirable error in refraction (0.05 arcsec) is indicated. All calculations have been made at the zenith distance of $60^{\circ}$

tral types earlier G0; and we should separate supergiants, giants and dwarfs for stars G5 and later to provide the accuracy of 0.02 arcsec per luminosity class parameter in calculated refraction. There are many spectral classification methods aimed at study of spacial distributions of stars in galactic investigations and therefore providing segregation of stars into luminosity classes. The above mentioned classification system of Malyuto et al. (1997) easily segregates $\mathrm{K}-\mathrm{M}$ dwarfs, giants and supergiants. The segregation of stars into luminosity classes is possible in the conventional MK system (Johnson \& Morgan 1953) and other similar ones (e.g. Upgren 1963; Seitter 1975; Houk 1994) as well as with the use of photometric methods (e.g. Straizys 1992). We conclude that the accuracy of 0.02 arcsec per luminosity class parameter in calculated refraction is achievable. If we are satisfied with the accuracy of 0.05 arcsec per luminosity class parameter in calculated refraction or lower, we would need spectral types only (luminosity effects become unsignificant).

If astrometric observations near the galactic plane are performed, interstellar reddening effects should be taken into account. In Fig. 1 and Fig. 2 the calculated reddening effects are shown for representative spectral types and $T_{\text {eff }}$, respectively, at different degrees of interstellar reddening. Stellar temperature and reddening effects in refraction are of the same order; for reddened stars the temperature effects are smaller than for unreddened stars. Table 3 contains the accuracies in interstellar reddening required to achieve the indicated accuracies in calculated refraction at 
Table 3. Accuracies in $E(B-V)$ required to achieve the desirable accuracies per parameter in calculated refraction for dwarfs

\begin{tabular}{l|rrrrr|l}
\hline $\begin{array}{l}\text { Accuracy in } \\
\text { refraction } \\
\text { [as] }\end{array}$ & 0.5 & 1 & 2 & 3 & 4 & \\
\hline 0.02 & 0.05 & 0.06 & 0.11 & 0.21 & 0.38 & \\
0.05 & 0.13 & 0.16 & 0.28 & 0.53 & 0.95 & B0 \\
0.10 & 0.26 & 0.32 & 0.56 & 1.06 & 1.90 & \\
\hline 0.02 & 0.07 & 0.09 & 0.17 & 0.31 & 0.51 & \\
0.05 & 0.18 & 0.24 & 0.42 & 0.77 & 1.28 & G0 \\
0.10 & 0.36 & 0.48 & 0.84 & 1.54 & 2.56 & \\
\hline 0.02 & 0.11 & 0.14 & 0.26 & 0.44 & 0.67 & \\
0.05 & 0.28 & 0.36 & 0.64 & 1.1 & 1.68 & M0 \\
0.10 & 0.56 & 0.72 & 1.28 & 2.2 & 3.36 & \\
\hline
\end{tabular}

different degrees of interstellar reddening for B0, G0 and M0 dwarfs. The data show that the most stringent requirement to accuracy in reddening is for earlier type stars. We see from the first column of Table 3 that we should achieve an accuracy of 0.05 in $E(B-V)$ for B0V star in order to provide an accuracy of 0.02 arcsec per parameter in refraction. The same conclusions have been made for other luminosity classes (giants and supergiants).

Recently Weaver \& Torres-Dodgen (1997) has described a classification system (based on $15 \AA$ resolution spectra obtained with silicon-based detector) which provides an accuracy of 0.4 spectral subtype and 0.05 in $E(B-V)$ for $\mathrm{A}$-stars. In more general case the parameter $E(B-V)$ is defined from the formulae

$E(B-V)=(B-V)-(B-V)_{0}$,

where $B-V$ is the observed index for a concrete star, $(B-V)_{0}$ is the intrinsic index for respective spectral type and luminosity class in the MK system. Schmidt-Kaler (1982) has estimated the intrinsic scatter for a given type being from \pm 0.02 to \pm 0.06 in $(B-V)_{0}$, the typical accuracy of the observed $(B-V)$ index is about 0.01 . We see from the equation 3 that, as a rule, accuracy of 0.05 in $E(B-V)$ may be realized if reliable MK spectral types and luminosity classes are available (examples of the respective spectral classification systems were given above). We conclude that the accuracy of 0.02 arcsec per reddening parameter in calculated refraction is achievable.

Combining the above results for stellar parameters used in refraction calculations (for spectral type, luminosity class, degree of interstellar reddening) we conclude that there are about the same achievable accuracies in refraction (about 0.02 arcsec per each stellar parameter). Therefore, the combined error in calculated refraction due to uncertainties in three stellar parameters discussed above is about $0.03-0.04 \operatorname{arcsec}$ (it is by a half order better than the error for the best available observed stellar positions).

Till now all authors had ignored the possible dependencies of atmospheric refraction on stellar metal abun-

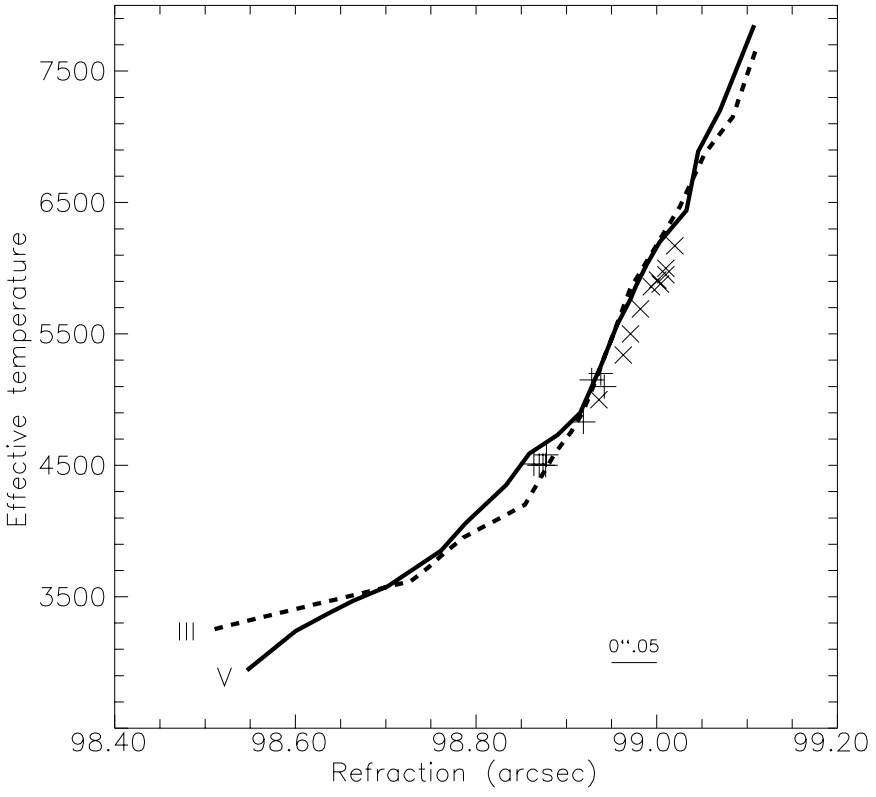

Fig. 4. The dependence of the calculated refraction on $T_{\text {eff }}$ for metal-deficient stars $(-1.4>[\mathrm{Fe} / \mathrm{H}]>-2.9)$. The designations used: plus signs - metal-deficient $\mathrm{G}-\mathrm{K}$ giants, crosses metal-deficient $\mathrm{F}-\mathrm{G}$ dwarfs. The lines are the same as in Fig. 3 (correspond to stars of normal metal abundance) but the calibration "Spectral type versus $T_{\text {eff" }}$ of Schmidt-Kaler (1982) is involved. One desirable error in refraction $(0.05$ arcsec $)$ is indicated. All calculations have been made at the zenith distance of $60^{\circ}$

dance (it is the third main physical parameter in spectral classification, side by side with spectral types and luminosity classes). To estimate it, we have added the available spectral energy distributions for selected metal-deficient stars (giants and dwarfs), as given by Sviderskiene (1992). The data were corrected for interstellar reddening. The values of the main physical parameters for these stars are taken from the catalogue of $[\mathrm{Fe} / \mathrm{H}]$ determinations (Cayrel de Strobel et al. 1997). For our estimates only the stars with the lowest metal abundance $(-1.4>[\mathrm{Fe} / \mathrm{H}]>-2.9)$ are discussed, the known spectral binaries are excluded. There are 9 metal-deficient $\mathrm{G}-\mathrm{K}$ giants and 11 metaldeficient $\mathrm{F}-\mathrm{G}$ dwarfs in our list. Because of the uncertainty of spectral types for such stars we have calculated and discussed the dependence of the calculated refraction only on $T_{\text {eff }}$ (Fig. 4). We conclude that the effect of metal abundance on refraction is small and reaches only $0.02-0.03$ arcsec in these extreme cases. The metaldeficient dwarfs ( $\mathrm{F}-\mathrm{G}$ subdwarfs) have slightly larger refraction than the dwarfs of normal metal abundance with the same temperature.

We have also analysed the dependencies of calculated refraction on atmospheric parameters (ground-based temperature, pressure, dew point, subsequently). From the slopes of these dependencies we have estimated how high the errors per every parameter in calculated refraction should be to achieve desirable accuracies of $0.01,0.02$, 
Table 4. Accuracies in atmospheric parameters necessary to reach the desirable accuracies per parameter in calculated refraction

\begin{tabular}{l|rrr}
\hline $\begin{array}{l}\text { Accuracy in } \\
\begin{array}{l}\text { Refraction } \\
\text { as] }\end{array}\end{array}$ & $\begin{array}{r}\text { Accuracy in atmospheric parameters } \\
\text { Temperature } \\
\text { [degC] }\end{array}$ & $\begin{array}{r}\text { Pressure } \\
{[\mathrm{mm}]}\end{array}$ & $\begin{array}{r}\text { Dew point } \\
\text { [degC] }\end{array}$ \\
\hline 0.01 & 0.03 & 0.07 & 1.1 \\
0.02 & 0.06 & 0.15 & 2.3 \\
0.05 & 0.14 & 0.37 & 5.7 \\
0.10 & 0.29 & 0.75 & 11.5 \\
\hline
\end{tabular}

0.05 and 0.1 arcsec per input parameter in calculated refraction. The results are given in Table 4 and should be compared with the accuracies of measuring devices. According to Stone (1996), the atmospheric pressure measured with Setra digital barometers may be calibrated to $\pm 0.1 \mathrm{~mm}$, the dew point sensors used by Stone (1996) are accurate to $\pm 2^{\circ} \mathrm{F}$ (about $\pm 1^{\circ} \mathrm{C}$ ). From the comparison of the just mentioned accuracies with the data of Table 4 we conclude that the accuracies of about $0.01-0.02$ arcsec per every parameter in calculated refraction are achievable with these devices (the highest accuracy is achievable per dew point parameter).

But the temperature case is more complicated. Although the resistance temperature detector may be calibrated to $\pm 0.05^{\circ} \mathrm{C}$ (Stone 1996), "in general, the accuracy of measurements of the temperature cannot be forced to lower than $1^{\circ} \mathrm{C}$. The reason is not to be found in the measuring instruments but in local temperature gradients inside the dome and telescope itself" (Ploner 1996). The problem has been carefully studied by Stone et al. (1996), their observational stellar positions are corrected for so called room and tube refractions (with the use of some temperature probes giving the temperature distribution within the dome at any particular time, air circulation system is installed). The room refraction is found to be the most serious source of errors in calculated refraction (typically the error is about 0.03 arcsec but can exceed 0.1 arcsec on some nights). The refraction model used in the present paper doesn't take into account the room refraction. However, the respective temperature errors in calculated refraction may be avoided or radically reduced if we consider differential refraction only (see the next section).

\section{Differential calculations for atmospheric refraction}

If two or more stars are observed simultaneously within a frame of a CCD detector, differential measurements of stellar positions become possible. In differential calculations for refraction the respective uncertainties in atmospheric parameters become unimportant because ground-based atmospheric temperature, pressure and dew point are the same for every object at the time of the observation. We may expect that any changes in these parameters (as well as in temperature distribution within the dome) are small during an exposure. Then we should take into account only the color effects in calculated refraction due to differences in spectral types of stars and to different degrees of interstellar reddening. Judging on the results of the previous section, we conclude that the total error in calculated differential refraction should be within 0.04 arcsec if the best available spectral classification systems are applied.

In the subsequent analysis of differential refraction we follow mainly the pattern used by Gubler \& Tytler (1998) although the aim, method and details of the analysis are different. Regardless of which approach is used, the following quantities must be specified in order to perform the calculations:

$T_{1} \quad$ effective temperature of star 1 ,

$T_{2} \quad$ effective temperature of star 2 ,

$\Delta z_{0} \quad$ difference between the (observed) zenith distances of stars 1 and 2 ,

$z_{1} \quad$ observed zenith distance of star 1 ,

$A T, P, D$ ground-based atmospheric temperature, pressure and dew point at the time of the observation, respectively.

We present the results of calculations for one example: the differential refraction $\Delta R_{\mathrm{m}}$ between stars 1 and 2 for $z_{1}=60^{\circ}$ and $\Delta z_{0}=12$ arcmin has been calculated (taking into account that the frame of our CCD detector corresponds to the area of $12 \times 18$ arcmin on the sky). We use the same approach to calculations as in the previous section with the same typical set of ground-based atmospheric parameters. A knowledge of the exact values of atmospheric parameters is not crucial in this differential case: as an example, if we change the ground-based temperature (the most uncertain atmospheric parameter) by $2{ }^{\circ} \mathrm{C}$, the respective calculated differential refraction are changed less than by 0.005 arcsec in the most extreme cases (if one goes from spectral type $\mathrm{B}$ to $\mathrm{M}$ at a zenith distance of $60^{\circ}$ ). Keeping in mind the planned differential measurements of positions of solar system bodies relative to positions of reference stars, we take star 1 as a solar type star $(\mathrm{G} 2 \mathrm{~V})$, star 2 is $\mathrm{G} 2 \mathrm{~V}, \mathrm{~B} 0 \mathrm{~V}, \mathrm{M} 2 \mathrm{~V}, \mathrm{~B} 0 \mathrm{I}, \mathrm{M} 2 \mathrm{I}$ at different degrees of interstellar reddening $(0.0,0.5,1.0$, 2.0, 3.0 and 4.0) subsequently. The calculated differential positions are given in Table 5 .

Three effects are combined together in this table: one depends on separation of the stars along the zenith direction (separation effect) a second is influenced by the difference in spectral types of the stars (stellar temperature effect) and a third is influenced by degrees of interstellar reddening (interstellar reddening effect). The pure separation effect at different reddenings is given in the second column where star 2 is of the same spectral type $(\mathrm{G} 2 \mathrm{~V}$ ) as star 1. The total effect is largest for B0V (of order 1 arcsec) and decreasing with increasing the reddening. The appropriate observations for some pairs of 
Table 5. Differential positions $\Delta R_{\mathrm{m}}$ (star 2 minus star 1 ) given in arcsec. Star 1 is $\mathrm{G} 2 \mathrm{~V}$ observed at $z_{1}=60^{\circ}$, the calculated refraction is 98.977 arcsec. In this example star 2 is observed at $z_{2}=60^{\circ} 2$. Its $E(B-V)$ and spectral types are as given in this table

\begin{tabular}{l|rrrrr}
\hline$E(B-V)$ & \multicolumn{5}{|c}{ Differential positions $\Delta R_{\mathrm{m}}$} \\
for star 2 & G2V & B0V & M2V & B0I & M2I \\
\hline 0.0 & 0.796 & 1.099 & 0.520 & 1.074 & 0.489 \\
0.5 & 0.647 & 0.899 & 0.427 & 0.877 & 0.405 \\
1.0 & 0.526 & 0.726 & 0.356 & 0.709 & 0.340 \\
2.0 & 0.365 & 0.481 & 0.263 & 0.471 & 0.255 \\
3.0 & 0.277 & 0.347 & 0.211 & 0.342 & 0.206 \\
4.0 & 0.227 & 0.275 & 0.178 & 0.272 & 0.175 \\
\hline
\end{tabular}

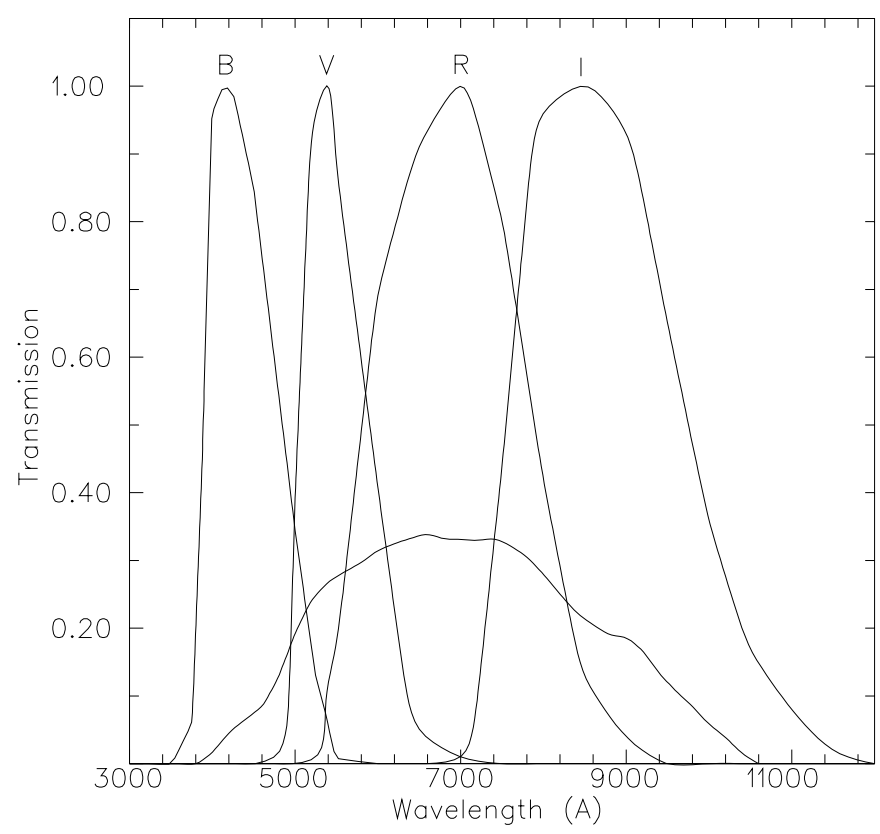

Fig. 5. The filter transmission curves together with the quantum efficiency (the lower line) of a CCD detector used in our study

stars with very differing spectral types taken from the Hipparcos catalogue are planned to compare calculated and true refraction.

\section{Refraction in connection with $B V R I$ photometry}

Many spectral and photometric classification methods (some examples were given above) provide MK spectral types and luminosity classes of stars. These data are necessary for accurate refraction calculations. As to photometric classification, it may only be realized with the use of filter combinations whose bands are narrower than $300-500 \AA$. However, photometries with band widths of the order of $1000 \AA$ and more are performed. They may reach fainter stars and are very useful for many tasks. The most wide-spread photometry is the $U B V R I$ photometry

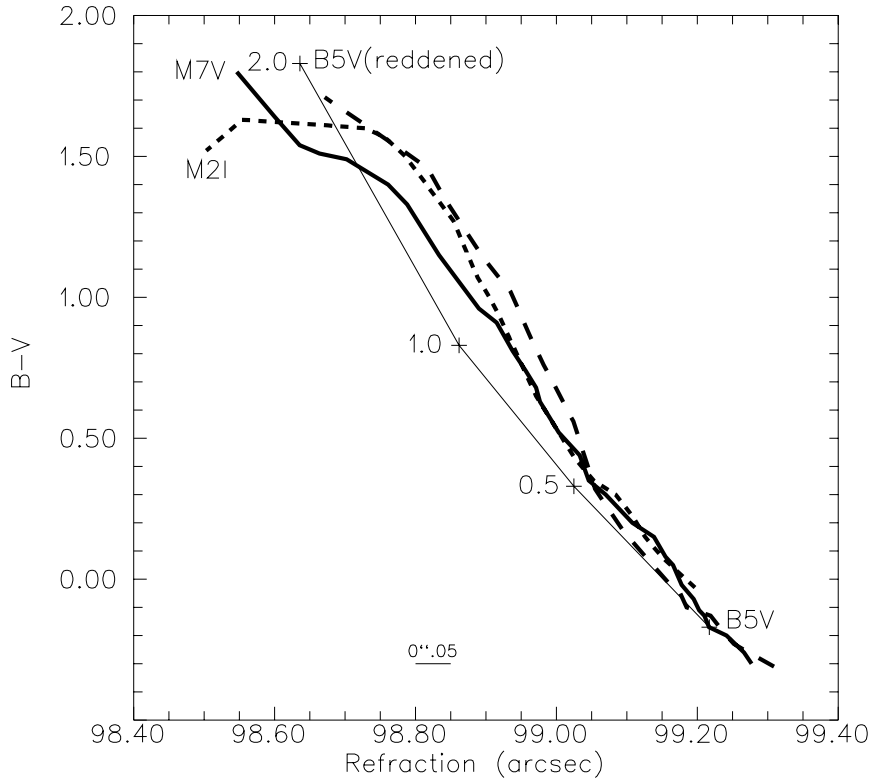

Fig. 6. The dependencies of the calculated refraction on $B-V$ indices for different luminosity classes. The thin line is the reddening line for B5 dwarf at different $E(B-V)$. Other designations are the same as in Fig. 3. All calculations have been made at the zenith distance of $60^{\circ}$

of Johnson (1965). In Fig. 5 their filter transmission curves are given together with the quantum efficiency of a CCD detector used in our study. Here we restrict ourselves with the $B V R I$ range where the CCD detector is sensitive.

To assign photometric indices to the tabulated spectral energy distributions for different spectral types and luminosity classes (Sviderskiene 1988) we use the mean relations of Schmidt-Kaler (1982) where the $B-V$ indices are given as functions of MK spectral types, separately for dwarfs (luminosity class V), giants (luminosity class III) and supergiants (luminosity class I), respectively. For $V-R$ and $V-I$ indices we take the respective relations from Straizys (1992). In Figs. 6, 7 and 8 the dependencies of the calculated refraction on $B-V, V-R$ and $V-I$ indices respectively are given for different luminosity classes, one accuracy of refraction measurements ( 0.05 arcsec) is indicated. We conclude from these figures that in the case of the $B-V$ index the luminosity effects are significant for $\mathrm{K}-\mathrm{M}$ types; but for $V-R$ and $V-I$ indices these effects are small in comparison with the indicated accuracy of refraction measurements.

In Figs. 6, 7 and 8 the interstellar reddening effects are also given for B5 dwarf at different degrees of reddening. To get the $E(V-R)$ and $E(V-I)$ values for the corresponding $E(B-V)$, the following reddening ratios taken from Taylor (1986) are used:

$E(R-I) / E(B-V)=0.838$

$E(V-R) / E(B-V)=0.80$.

These ratios are valid for stars earlier than A0. In the case of $B-V$ indices (Fig. 6) the reddening line is deviating 


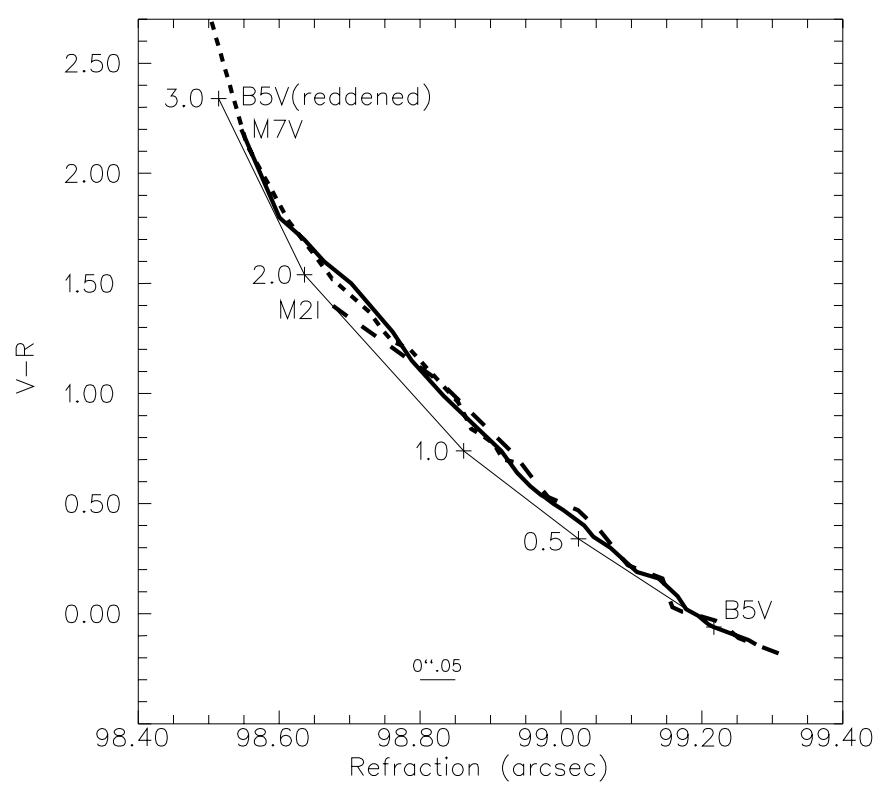

Fig. 7. The dependencies of the calculated refraction on $V-R$ indices for different luminosity classes. The designations are the same as in Fig. 6. All calculations have been made at the zenith distance of $60^{\circ}$

significantly from the lines for unreddened dwarfs. The situation is quite different for $V-R$ and $V-I$ indices (Figs. 7 and 8 , respectively) where the reddening lines are almost parallel or nearly coincide with the lines for unreddened stars. Judging on the last two figures we may suspect that there is no necessity to know $E(B-V)$ as well as spectral types and luminosity classes in order to obtain the refraction from $V-R$ or $V-I$ indices with accuracies of $0.03-0.05$ arcsec in refraction.

In this respect it is to the point to quote a remark of Straizys (1992) that in case of color indices at $\lambda \leq$ $5000 \AA$ (it is close to the range of the CCD detector sensitivity) luminosity effects are small; reddening and temperature effects coincide. As a result, atmospheric extiction coefficient (discussed by Straizys) may be expressed by one linear function of color index. Similarily, refraction may be expressed by about a one-to-one function of $V-R$ (or $V-I$ ) index for all spectral types, luminosity classes and reddenings. The $V-I$ index seems to be the most preferable parameter for estimating refraction because at least for B5 dwarf the reddening line is almost non-distinguishable from the line for unreddened stars in Fig. 8 and the interval of measurements is large in comparison with accuracy of measurements (about 0.02 in $V-I$, Johnson et al. 1966).

To get more examples, we have also calculated the reddening line position for B5 supergiant in Fig. 8 and found that this line is also almost non-distinguishable from the respective line for B5 dwarf. We conclude that the possibility may exist to estimate mean refraction for a star from $V-I$ index only with the accuracy of 0.05 arcsec or better;

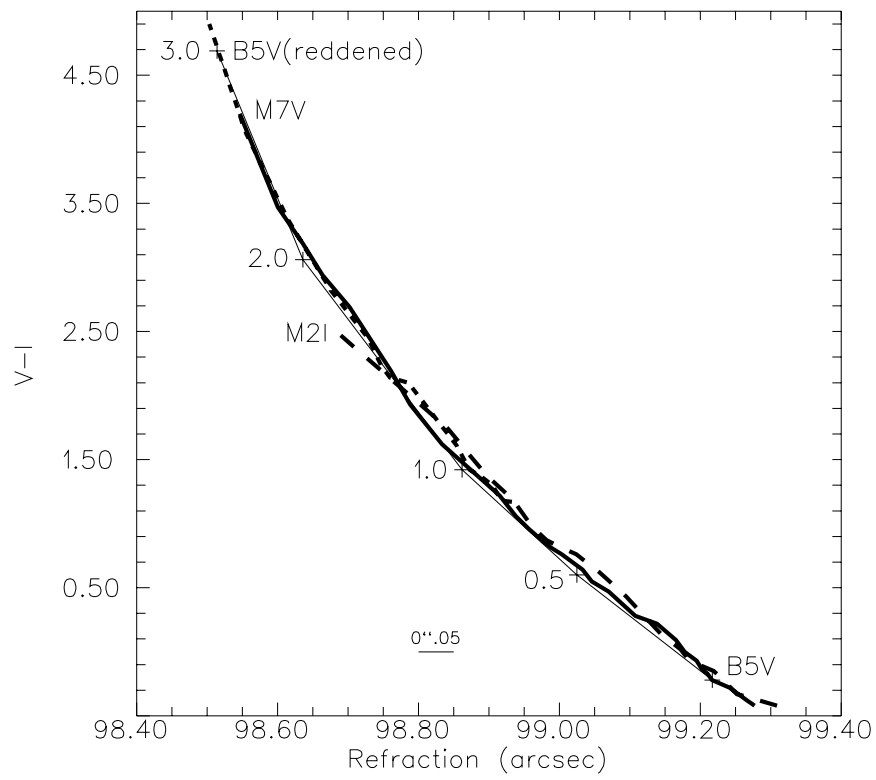

Fig. 8. The dependencies of the calculated refraction on $V-I$ indices for different luminosity classes. The designations are the same as in Fig. 6. All calculations have been made at the zenith distance of $60^{\circ}$

spectral type, luminosity class and interstellar reddening may be unknown. The $V-I$ index is well known as the measure of $T_{\text {eff }}$ (Straizys 1992); in addition, its unsensitivity to metal abundance effects at least for F - G dwarfs has been detected (Malyuto 1969; Alonso et al. 1996).

The most correct approach to attack this problem is to calculate synthetic indices (by convolution of tabulated spectral energy distributions with the transmittance functions of $B V R I$ filters) for different spectral types and luminosity classes and at different artificial reddenings. It should be compared with the calculated refraction in order to check the conclusion of present Section and to extend it to later spectral type intervals.

\section{Conclusions}

The Stone (1996) code calculating mean refraction has been adjusted to the case of the $10^{\prime \prime}$ LX200 telescope installed at the Lohrmann Observatory in Dresden. We have estimated how accurate the appropriate stellar and atmospheric parameters should be to provide desirable accuracies in calculated refraction. The conclusion has been made that the accuracy of calculated refraction of 0.02 arcsec per one stellar parameter (spectral type, luminosity class, degree of interstellar reddening) should be achievable if the best available spectral classification systems are applied. The special case of computing differential refraction has also been discussed. The usefulness of photometric $V-R$ and $V-I$ indices in estimating mean refraction has been justified. 
The dependence of atmospheric refraction on stellar metal abundance (it is the third parameter in spectral classification, side by side with spectral types and luminosity classes) has been estimated. We have found that the effect of metal abundance does not exceed $0.02-$ 0.03 arcsec in calculated refraction.

We underline that accurate spectral classification of stars or color photometry should accompany any exact determinations of stellar positions. The use of objective prisms in spectral classification seems to be very efficient. Objective prism camera is the most efficient stellar spectrograph, because it makes use of the full telescope field. No light losses occur at a slit and those in the optic are small in comparison to slit spectrographs. The available classification methods based on photographic objective prism spectra can equally well be applied to CCD objective prism images. Some specific problems will arise because of the use of non-coinciding wavelength ranges but much higher accuracy and quantum efficiency parallel with linear energy response of CCD detectors are large gains.

In the above discussion we have referenced many classification methods. They were developed for different tasks and the used resolution as well as penetrating abilities are very differing. The problem of choosing optimal spectral resolution of objective prism CCD images aimed at a concrete spectral classification task should be set up. Under optimal resolution we mean the resolution which is as low as possible (to reach fainter stars) but which yet allows to perform a spectral classification with the accuracy required for the concrete task. The idea is to take one sample of stars observed with one relatively high spectral resolution, to classify these stars with a chosen classification method and to estimate the respective classification accuracy. Then the spectral resolution should artificially be decreased step by step and at every stage the accuracies of spectral classification should be estimated. We should stop the process at the stage when further deterioration of spectral resolution makes classification accuracy too bad for any reasonable classification task. The spectral resolution at the stage when the classification accuracy is yet good enough for the concrete classification task, should be considered to be optimal for this task. The results of this investigation can be applied to some astrometric, astrophysical and galactic studies.

Acknowledgements. We are very grateful to Ronald C. Stone of the U.S. Naval Observatory for sending us his code developed for computing refraction as well as for encouraging comments what made this investigation possible. We are thankful to a referee for his careful reading of the paper and for helpful suggestions. We are very grateful to Prof. M. Soffel who has suggested the idea of the present investigation and to Dr. K. Kurzynska for comments on this paper.

\section{References}

Allen C.W., 1973, Astrophysical Quantities, Univ. of London. The Athlone Press

Alonso A., Arribas S., Martinez-Roger C., 1996, A\&A 313, 873

Cayrel R., Perrin M.-N., Barbuy B., Buser R., 1991, A\&A 247, 108

Cayrel de Strobel G., Soubiran C., Friel E.D., Ralite N., Francois P., 1997, A\&AS 124, 299

Johnson H.L., 1965, ApJ 141, 923

Johnson H.L., Mitchell R.I., Iriarte B., Wisniewski W.Z., 1966, Comm. Lunar Planetary Lab. 4, 99

Johnson H.L., Morgan W.W., 1953, ApJ 117, 313

Gubler J., Tytler D., 1998, PASP 110, 738

Houck N., 1994, ASP Conf. Ser. 60, 285

Katz D., Soubiran C., Cayrel R., Adda M., Cautain R., 1998, A\&A 338, 151

Kovalevsky J., 2000, The Next Decades: A New Boost to Astrometry? In: Journées 1999 \& IX. LohrmannKolloquium "Motion of Celestial Bodies, Astrometry and Astronomical Reference Frames", Soffel M., Captaine N. (eds.) (in press)

Malyuto V., 1969, Publ. Tartu Obs. 38, 79

Malyuto V., Oestreicher M., Schmidt-Kaler Th., 1997, MNRAS 286,500

Malyuto V., Schmidt-Kaler Th., 1997, A\&A 325, 693

Nandy, et al., 1977, MNRAS 178, 63

Owens J.C., 1967, Appl. Opt. 6, 51

Pickles A.J., 1998, PASP 110, 863

Ploner M., 1996, Geowissenschaftlische Mitteilungen 46, Technische Universität Wien

Schmidt-Kaler Th., 1982, in: Landolt-Börnstein, New Series, Vol. VI/ab. Springer, Berlin, Heideberg, New York, p. 17

Schildknecht T., 1994, Geodätisch-Geophysikalische Arbeiten in der Schweiz 49, 1

Seitter W.C., 1975, Atlas for Objective Prism Spectra, Bonner Spectral Atlas II. Ferd. Dümmler Verlag, Bonn

Soubiran C., Katz D., Cayrel R., 1998, A\&AS 133, 221

Stone R.C., 1984, A\&A 138, 275

Stone R.C., 1996, PASP 108, 1051

Stone R.C., Monet D.G., Monet A.K.B., Walker R.L., Ables H.D., Bird A.R., Harris F.H., 1996, AJ 111, 1721

Straizys V., 1992, Multicolor Stellar Photometry. Pachart Publishing House, Tucson

Straizys V., Liubertas R., Lazauskaite R., 1998, Baltic Astron. 7,529

Sviderskiene Z., 1988, Bull. Viln. Astron. Obs. 35

Sviderskiene Z., 1992, Bull. Viln. Astron. Obs. 86

Taylor B.J., 1986, ApJS 60, 577

Upgren A.R., 1962, AJ 67, 37

Upgren A.R., 1963, AJ 68, 194

Weaver Wm.B., Torres-Dodgen A.V., 1995, ApJ 446, 300

Weaver Wm.B., Torres-Dodgen A.V., 1997, ApJ 487, 847 